\title{
Hybrid simulation for teaching clinical breast examination (CBE) for physicians in training
}

\author{
Rana Sharara-Chami, Umayya Musharrafieh, Anwar Nassar, Zavi Lakissian and Joseph Nassif* \\ Department of Obstetrics and Gynecology, American University of Beirut Medical Center, Lebanon
}

\begin{abstract}
Introduction and background: Breast cancer has become a major national and international health issue; recommendations for clinical breast examination ( $\mathrm{CBE}$ ) are lacking compared to successful campaigns for mammography screening. Clinical breast examination is a traditionally difficult portion of the physical examination to teach and to master. Residents are often taught by didactic lectures and low fidelity breast models with or without the opportunity to practice on real patients based on their availability and willingness to be examined by a resident.

Methods: This study aims to assess hybrid simulation as a complimentary teaching method to CBE to first- and second-year obstetrics and gynecology and family medicine residents. Traditionally, and prior to our intervention, residents received CBE teaching via a didactic lecture and they practiced on a low-fidelity table-top single silicone breast model (MammaCare $\left.{ }^{\circledR}\right)$.

Our intervention comprises of the introduction of a standardized patient (SP) wearing a silicone breast jacket (Limbs \& Things ${ }^{\circledR}$ ). During the 2 weeks following the didactic lecture, residents got the chance to deliberately practice $\mathrm{CBE}$ on the table-top model. In the beginning of the 3rd week, a CBE session (CBE I) was scheduled with an SP wearing the silicone breast jacket with one-on-one feedback by one of the investigator physicians (UM and JN). Prior to giving the feedback, residents were evaluated by the physicians and the SP. In the following 2 weeks, residents had 3 practice sessions with the SP wearing the breast jacket to improve on their skills based on the feedback provided to them. After the completion of the 4-week practice period, residents underwent an Objective Structured Clinical Examination (OSCE) (CBEII) where they examined an SP wearing a breast jacket with different pathologies. Assessment included a subjective evaluation by the resident regarding their confidence and readiness to complete the $\mathrm{CBE}$, an objective assessment by the SP, and assessments performed by three clinicians who independently evaluated videos of the OSCE encounter.
\end{abstract}

\section{Objectives}

1. To assess the effectiveness of hybrid simulation in teaching CBE to residents.

2. To improve the quality of teaching of an important and sensitive component of physical examination to future practitioners.

3. To improve residents' ability to detect early breast cancer through the use of simulation models.

\section{Introduction and background}

Breast cancer remains the most common cancer among women worldwide [1]. Globally, preventive efforts such as raising awareness and advocating for regular breast examinations are aimed towards early detection. Clinical Breast Examination (CBE) is extensively practiced in countries like the United States and is considered part of the regular health exam [2]. Although the evidence to support the benefit of breast examination in terms of reducing breast cancer morbidity and mortality via early breast cancer detection is not well established [2], CBE remains a basic required skill for physicians in training and offers healthcare providers the opportunity to educate women on breast health.

According to the American College of Surgeons, overcoming the barriers to $\mathrm{CBE}$ performance starts with proper training. Didactic presentations, visual demonstrations and practical sessions that provide feedback, all constitute the components of proper training [2]. Even standardized patients alone have proven efficacious in instructing $\mathrm{CBE}$ to healthcare providers $[3,4]$; other studies have shown that simulators could have a role in relieving performance anxiety among physicians in training [5]. A study on the pelvic examination simulation model found that the addition of a standardized patient, albeit separately from the participants' interaction with the simulator, improved learning outcomes [6]. Additionally, two independent trials found that CBE simulators produced significant gains in clinical breast examination skills suggesting that this technology may improve the accuracy and quality of breast cancer screening [7].

Our goal for this study is to assess hybrid simulation as an alternative method for teaching CBE to residents by incorporating a patient actress i.e. a trained, standardized patient, wearing a breast simulator jacket (Appendix A). This could ultimately improve the quality of teaching

${ }^{\star}$ Correspondence to: Minimally Invasive Gynecological Surgery Endometriosis, Department of Obstetrics and Gynecology American University of Beirut Medical Center, Middle East Society of Gynecological Endoscopy (MESGE), Lebanon, E-mail: jn25@aub.edu.lb

Received: January 21, 2019; Accepted: January 28, 2019; Published: January 31, 2019 
an important and sensitive component of physical examination to future practitioners. We hypothesize that the combination of breast simulators and standardized patients in a single encounter will prove an effective method for teaching CBE, particularly in terms of lesion detection and identification.

\section{Significance}

It is a crucial task for medical education leaders to ensure that physicians-in-training are proficient and competent in CBE. Currently, there is no evidence in the literature on the effectiveness of hybrid simulation in teaching CBE to residents, nor studies that focus on assessing the educational experience of residents while performing CBE. A similar study [8] was conducted by the principle investigator which found that hybrid simulation improved lesion identification $(\mathrm{p}<0.001)$ and relieved the fear of missing a lesion on CBE $(\mathrm{p}=0.043)$ and increased satisfaction with the teaching method among medical students $(p=0.002)$ [8]. This study is the first of its kind to use modern techniques to elevate the proficiency of residents' CBE skills and make the teaching of CBE to residents, and potentially the detection of breast cancer lesions, more rounded and even more effective.

\section{Methods and evaluation}

The study is a quality improvement project and an assessment of a new teaching method.

\section{Study participants}

1. First and Second Year (PGY I-II) Obstetrics and Gynecology Residents $(\mathrm{N}=12)$

\section{First and Second Year (PGY I-II) Family Medicine Residents ( $\mathrm{N}=12)$}

$\mathrm{CBE}$ is a skill taught during medical school and revisited during the first two years of residency. This participant pool reflected the number of PGY I-IIs admitted each year to the above-mentioned departments; therefore, it represented a convenient sample. All 24 residents from these two departments took part in the didactic lecture and traditional practice on the table-top breast model as part of their curricular requirements. The residents were notified by their respective departments via e-mail of the lecture on CBE, and residents' attendance was mandatory. The lecture was given for both departments by JN. Following the lecture ZL explained and discussed the research aspects of the practicums and the OSCE to the residents and asked for their consent to use the data collected throughout the course of these interventions for research purposes. All residents present at the lecture were afforded the opportunity to participate in the practicums and the OSCE whether they consented to the use of their data for research or not. Ten residents consented, 4 from family medicine and 6 from obstetrics and gynecology.

In the 4 weeks between the lecture and the OSCE, residents were encouraged to practice: In the first 2 weeks on the low-fidelity table-top single silicone breast model (MammaCare ${ }^{\circ}$ ) and in the last 2 weeks, during 3 practice sessions with an SP wearing the breast jacket. Midway through the practice period, a CBE session (CBE I) with a SP + Breast Jacket was organized for all residents in the presence of JN and UM. Residents were asked to perform a full CBE on a standardized patient wearing a breast simulator jacket (Limbs \& Things ${ }^{\oplus}$ ) (see Simulators in Appendix A) and they were evaluated by the SP and, JN and UM, prior to receiving feedback on their performance.
OSCEs are now an established assessment and training method for residents and students in both departments. The OSCE encounter included a complete CBE examination with an SP wearing the breast jacket. The assessment of the OSCE included both objective (rated by the SP and 3 clinicians) and subjective evaluation by the resident, including: (1) Clinical Competency and completeness of the CBE examination and (2) Confidence and readiness to perform the CBE, respectively (Appendices B-F).

> The primary outcomes of CBE proficiency are CBE completeness and lesion identification.

> Secondary outcomes included self-reported performance and attitude during the CBE OSCE, including the resident's reported unease during $\mathrm{CBE}$ and its causes as well as their satisfaction with the teaching activity.

The OSCEs were recorded per standard practice. The videos were independently evaluated by 3 clinicians retrospectively to assess the residents' performance of the $\mathrm{CBE}$ and their ability in identifying lesions (Appendix B). To alleviate potential bias, the 3 clinicians were blinded to the specialty and year in training of the residents being evaluated.

\section{Standardized patients}

Standardized patients were selected from the American University of Beirut Medical School Standardized Patient Program. This program follows official institutional protocols for hiring SPs to participate in student teaching and assessment sessions. SPs were selected by the principle investigator (RSC) and UM for the practicum and OSCE based on the SPs previous experience and criteria relevant to the study.

SP characteristics: SPs are defined as individuals instructed to simulate patients in specific scenarios in order to evaluate learners' clinical and communication skills. For this assessment, SPs will represent middle-aged Lebanese women.

Training and measures: SPs were trained by the principle investigator and by AN and JN on their role and how to assess breast palpation on the simulator jacket. At the end of each encounter, the SP was also trained on how to evaluate the participant's CBE performance by scoring a checklist on CBE completeness. For quality assurance, the same SPs were used as during the previous study performed by the same group on students [8]; during that study, the investigators randomly audited the OSCE sessions to make sure that SPs were evaluating properly and correctly. The checklist included items on the technique of examination in both its visual inspection and palpation aspects.

At the end of each encounter, the resident filled an in-clinic note and a self-reported performance survey. The in-clinic note described their findings on the CBE, as well as a self-evaluation survey on their experience, confidence and readiness while performing the CBE.

\section{Ethical considerations}

The study was approved by the Institutional review board of the American University of Beirut-Medical Center. 


\section{Timeline}

- Fesidents were
recurited
- Consent was obtained
(N=10)
table-top single breast

\section{Results}

Four family medicine and 6 obstetrics and gynecology residents participated in the study. Fifty percent were male, $80 \%$ were older than 25 years of age and 90\% spoke Arabic as their native language. On average, residents had performed $\mathrm{M}=3.67$ (SD 1.15) CBEs on actual patients.

Residents completed a pre- and post-OSCE self-evaluation to assess their confidence with performing the CBE, detecting a lesion and communicating with the patient (Tables 1 and 2). In both pre-and post-evaluations, $90 \%$ of residents were comfortable performing a CBE, identifying a lesion, and were confident about their communication skills while performing the CBE. Most residents felt uneasy about the possibility of missing a lesion, yet all of them detected, identified and noted the lesions correctly.

Percent agreement among the evaluators (RSC, UM, JN and the SPs) was $86 \%$. Overall, residents scored 19.80 (3.3) over a maximum score of 24 when they performed a CBE following the didactic lecture, and a slightly improved score of 20.20 (4.6) when they performed the OSCE after 4 weeks of practice ( $p$.759) (Table 3). They scored 4.2 (1.6) on visual inspection during the first $\mathrm{CBE}$ and 4.7 (2) on the OSCE ( $p$.495) over a maximum score of 6 . On palpation skills, residents scored $12(1.7)$ and 11.8 (2.1) on the first and second CBE evaluations respectively ( $p$.735) over a total of 14 points. Lastly, in the communications domain (scored over 4 ), scores were $3.6(0.5)$ and 3.5 $(1)$ in the first and second CBEs respectively $(p .798)$. All participants found the experience to be valuable, realistic and beneficial to their future practice and preparedness for CBE (Table 4).

\section{Discussion}

The high overall CBE completeness results, following the didactic lecture and practice, show that residents are quite proficient in performing the examination, particularly as most have had experience examining actual patients during their residency. This is affirmed by the participants' self-stated confidence and comfort in performing the examination. Yet, despite the high scores, residents were satisfied with the practical value and benefits of the simulated experience offered.

Lesion identification has been proven to be similar in students who learned on standardized patients (real breast tissue) and those who learned on a simulated model; hybrid simulation had an added value over low-fidelity simulation and non-guaranteed real patient encounters [9]. A major cause of CBE-related discomfort in medical students had been found to be the fear of missing a lesion on a real patient [5]. Similarly, this presented a unanimous cause for unease among our residents as stated in their responses in the pre-and postintervention self-assessment. Perhaps it is prudent to not assuage this unease completely, lest physicians become complacent in their guaranteed ability to detect a lesion.

In line with our findings, it had been previously shown that the use of simulators in teaching CBE, in addition to lectures, improves satisfaction among both teachers and learners. The participants' recommendation to adopt this method in curricula reflects the value of the practical teaching session of CBE despite the residents' proficient performance.

The study was conducted in a single center and included a small number of participants. A larger-scale study may lead to statistically significant results concurrent with our findings. Nonetheless, the fact that the CBE completeness scores were quite high and similar between the first $\mathrm{CBE}$ and the second is suggestive that $\mathrm{CBE}$ practicums on hybrid simulation after practicing on table-top models may not be a useful teaching method for residents. Residents are well familiar with the theory and have had real-patient encounters. Despite the cost associated with procuring the breast jackets, hybrid simulation could possibly replace practicing on a table-top model altogether. Hybrid simulation provides realism, higher satisfaction with the learning experience and could potentially be used to assess residents' communication skills around this sensitive part of the physical exam. This becomes especially true when patient availability and willingness is not guaranteed.

\section{Conclusion}

We employed hybrid simulation with standardized patients wearing breast jackets for residents to learn and practice CBE. Unlike with medical students [8], the simulation did not impart clinical knowledge, yet residents positively rated the teaching experience, and all agreed that it offered an opportunity to practice and improve their skills. Their recommendations for the inclusion of hybrid simulation in curricula shows their appreciation of the added value of this exercise to practice a sensitive yet important clinical examination, knowing patient encounters are not guaranteed, even during residency. 
Table 1. Pre-OSCE Resident Self Evaluation

\begin{tabular}{|c|c|c|c|c|}
\hline & Totally Disagree & Disagree & Agree & Totally Agree \\
\hline \multicolumn{5}{|l|}{ I am confident } \\
\hline performing the $\mathrm{CBE}$ & & $1(10 \%)$ & $8(80 \%)$ & $1(10 \%)$ \\
\hline identifying a lesion & & $2(20 \%)$ & $8(80 \%)$ & \\
\hline of my communication skills during a $\mathrm{CBE}$ & & & $8(80 \%)$ & $2(20 \%)$ \\
\hline my cultural competence in communicating with patients & & $1(10 \%)$ & $6(60 \%)$ & $1(10 \%)$ \\
\hline I feel comfortable & Not at all & A little & Quite a bit & Very much \\
\hline with performing the $\mathrm{CBE}$ & & & $8(80 \%)$ & $1(10 \%)$ \\
\hline communicating with the patient during $\mathrm{CBE}$ & & & $8(80 \%)$ & $1(10 \%)$ \\
\hline \multicolumn{5}{|l|}{ I feel comfortable with the following specific aspects of CBE } \\
\hline overall comfort & & & $8(80 \%)$ & $2(20 \%)$ \\
\hline visual inspection of the breast & & $1(10 \%)$ & $5(50 \%)$ & $4(40 \%)$ \\
\hline eliciting nipple discharge & & $2(20 \%)$ & $5(50 \%)$ & $3(30 \%)$ \\
\hline detecting abnormalities & & $1(10 \%)$ & $7(70 \%)$ & $2(20 \%)$ \\
\hline current level of knowledge, experience and training in $\mathrm{CBE}$ & & $2(20 \%)$ & $6(60 \%)$ & $2(20 \%)$ \\
\hline Causes of unease in learning and practicing CBE & Totally Disagree & Disagree & Agree & Totally Agree \\
\hline no anxieties & $2(20 \%)$ & & $5(50 \%)$ & $3(30 \%)$ \\
\hline possibility of missing a lesion & & & $7(70 \%)$ & $3(30 \%)$ \\
\hline intimate/personal nature of the examination & $2(20 \%)$ & $2(20 \%)$ & $5(50 \%)$ & $1(10 \%)$ \\
\hline causing harm or pain to the patient & $3(30 \%)$ & $2(20 \%)$ & $3(30 \%)$ & $2(20 \%)$ \\
\hline nipple and areola palpation & $2(20 \%)$ & $3(30 \%)$ & $4(40 \%)$ & $1(10 \%)$ \\
\hline communicating effectively with the patient & $3(30 \%)$ & $3(30 \%)$ & $3(30 \%)$ & $1(10 \%)$ \\
\hline general performance anxiety & $3(30 \%)$ & $2(20 \%)$ & $4(40 \%)$ & $1(10 \%)$ \\
\hline possible cultural dissimilarity between you and the patient & $3(30 \%)$ & $2(20 \%)$ & $4(40 \%)$ & $1(10 \%)$ \\
\hline
\end{tabular}

Table 2. Post-OSCE Resident Self Evaluation

\begin{tabular}{|c|c|c|c|c|}
\hline & Totally Disagree & Disagree & Agree & Totally Agree \\
\hline \multicolumn{5}{|l|}{ I am confident } \\
\hline performing the $\mathrm{CBE}$ & & & $6(60 \%)$ & $4(40 \%)$ \\
\hline identifying a lesion & & $1(10 \%)$ & $6(60 \%)$ & $3(30 \%)$ \\
\hline \multicolumn{2}{|l|}{ of my communication skills during a CBE } & $1(10 \%)$ & $5(50 \%)$ & $4(40 \%)$ \\
\hline \multicolumn{3}{|l|}{ my cultural competence in communicating with patients } & $6(60 \%)$ & $4(40 \%)$ \\
\hline I feel comfortable & Not at all & A little & Quite a bit & Very much \\
\hline \multicolumn{2}{|l|}{ with performing the $\mathrm{CBE}$} & & $5(50 \%)$ & $5(50 \%)$ \\
\hline \multicolumn{2}{|l|}{ communicating with the patient during $\mathrm{CBE}$} & $1(10 \%)$ & $6(60 \%)$ & $3(30 \%)$ \\
\hline \multicolumn{5}{|l|}{ I feel comfortable with the following specific aspects of CBE } \\
\hline overall comfort & & $1(10 \%)$ & $6(60 \%)$ & $3(30 \%)$ \\
\hline \multicolumn{2}{|l|}{ visual inspection of the breast } & $1(10 \%)$ & $4(40 \%)$ & $5(50 \%)$ \\
\hline \multicolumn{2}{|l|}{ eliciting nipple discharge } & & $5(50 \%)$ & $5(50 \%)$ \\
\hline \multicolumn{2}{|l|}{ detecting abnormalities } & $2(20 \%)$ & $6(60 \%)$ & $2(20 \%)$ \\
\hline \multicolumn{2}{|l|}{ current level of knowledge, experience and training in CBE } & $3(30 \%)$ & $5(50 \%)$ & $2(20 \%)$ \\
\hline Causes of unease in learning and practicing CBE & Totally Disagree & Disagree & Agree & Totally Agree \\
\hline no anxieties & $1(10 \%)$ & & $6(60 \%)$ & $2(20 \%)$ \\
\hline \multicolumn{2}{|l|}{ possibility of missing a lesion } & $1(10 \%)$ & $6(60 \%)$ & $3(30 \%)$ \\
\hline intimate/personal nature of the examination & $3(30 \%)$ & $1(10 \%)$ & $5(50 \%)$ & $1(10 \%)$ \\
\hline causing harm or pain to the patient & $4(40 \%)$ & & $4(40 \%)$ & $2(20 \%)$ \\
\hline nipple and areola palpation & $2(20 \%)$ & $3(30 \%)$ & $4(40 \%)$ & $1(10 \%)$ \\
\hline communicating effectively with the patient & $3(30 \%)$ & $3(30 \%)$ & $3(30 \%)$ & $1(10 \%)$ \\
\hline general performance anxiety & $1(10 \%)$ & $4(40 \%)$ & $3(30 \%)$ & $2(20 \%)$ \\
\hline possible cultural dissimilarity between you and the patient & $3(30 \%)$ & $2(20 \%)$ & $4(40 \%)$ & $1(10 \%)$ \\
\hline
\end{tabular}

Table 3. CBE Evaluation

\begin{tabular}{|c|c|c|c|}
\hline & CBE I (N=10) & CBE II (N=10) \\
\hline CBE completeness score (/24) & $19.80(3.3)$ & $20.20(4.6)$ \\
\hline Visual inspection score (/6) & $4.2(1.6)$ & $4.7(2)$ \\
\hline Palpation score (/14) & $12(1.7)$ & $11.8(2.1)$ \\
\hline Communication (/4) & $3.6(0.5)$ & 0.759 \\
\hline
\end{tabular}


Table 4. Evaluation of Teaching Method

\begin{tabular}{|c|c|c|c|c|}
\hline & Totally Disagree & Disagree & Agree & Totally Agree \\
\hline The simulations constituted a valuable learning experience & & & $8(80 \%)$ & $2(20 \%)$ \\
\hline The exercise provided a chance to learn in a safe environment & & $1(10 \%)$ & $5(50 \%)$ & $4(40 \%)$ \\
\hline The experience was realistic & & & $9(90 \%)$ & $1(10 \%)$ \\
\hline The exercise was effective in helping me integrate theory and practice & $1(10 \%)$ & $3(30 \%)$ & $4(40 \%)$ & $2(20 \%)$ \\
\hline The session should become a regular part of medical students' education & $1(10 \%)$ & $2(20 \%)$ & $4(40 \%)$ & $3(30 \%)$ \\
\hline The exercise was a worthwhile use of my time & & $2(20 \%)$ & $7(70 \%)$ & $1(10 \%)$ \\
\hline The exercise had no added value to the learning experience & $3(30 \%)$ & & $4(40 \%)$ & $3(30 \%)$ \\
\hline I feel more prepared for an upcoming CBE & & & $7(70 \%)$ & $3(30 \%)$ \\
\hline
\end{tabular}

\section{References}

1. Miller AB (2010) Screening for breast cancer in the Eastern Mediterranean Region. East Mediterr Health J 16: 1022-1024. [Crossref]

2. Saslow D, Hannan J, Osuch J, Alciati MH, Baines C, et al. (2004) Clinical Breas Examination: Practical Recommendations for Optimizing Performance and Reporting. CA Cancer J Clin 54: 327-344.

3. Coleman EA, Stewart CB, Wilson S, Cantrell MJ, O'Sullivan P, et al. (2004) An evaluation of standardized patients in improving clinical breast examinations for military women. Cancer Nurs 27: 474-482.

4. Costanza ME, Luckmann R, Quirk ME, Clemow L, White MJ, et al. (1999) The effectiveness of using standardized patients to improve community physician skills in mammography counseling and clinical breast exam. Prev Med 29: 241-248.
5. Pugh CM, Salud LH (2007) Fear of missing a lesion: use of simulated breast models to decrease student anxiety when learning clinical breast examinations. Am J Surg 193 766-770

6. Dilaveri C, Szostek J, Wang A, Cook D (2013) Simulation training for breast and pelvic physical examination: a systematic review and meta-analysis. BJOG Int J Obstet Gynaecol 120: 1171-1182. [Crossref]

7. Goldstein MK, Mehn MA, Pennypacker HS, Brost BC, Petersen WO, et al. (2012) Improved Clinical Breaser Examination Competencies VIa Intelligent Simulator Training. Meeting of the German Society for Senologie, Stuttgart.

8. Nassif J, Sleiman A, Nassar A, Naamani S, Sharara-Chami R (2016) Hybrid Simulation in Teaching Clinical Breast Examination, MedEdPublish.

9. Electronic Code of Federal Regulations, US Government Publishing Office.

Copyright: (C2019 Sharara-Chami R. This is an open-access article distributed under the terms of the Creative Commons Attribution License, which permits unrestricted use, distribution, and reproduction in any medium, provided the original author and source are credited. 